\title{
Learning curve and global benchmark values of laparoscopic sleeve gastrectomy: results of first 100 cases of a newly trained surgeon in an Italian center of excellence
}

\author{
Antonio Vitiello ${ }^{1}$ (D) Giovanna Berardi ${ }^{1} \cdot$ Nunzio Velotti $^{1} \cdot$ Vincenzo Schiavone $^{1} \cdot$ Mario Musella $^{1}$
}

Received: 18 February 2021 / Accepted: 20 June 2021 / Published online: 29 June 2021

(c) The Author(s) 2021

\begin{abstract}
To evaluate whether the learning curve for sleeve gastrectomy could be completed after 50 cases. First 100 patients undergoing LSG under a newly trained laparoscopic surgeon were included in this study and divided into two groups of 50 consecutive patients each. Perioperative outcomes were compared to recently introduced global benchmarks. Short-term weight loss was calculated as Total Weight Loss Percent (\%TWL) and complications were classified in accordance with the Clavien-Dindo classification. CUSUM analysis was performed for operative time and hospital stay. Mean preoperative age and BMI were $41.8 \pm 10.3$ years and $42.9 \pm 5.4 \mathrm{~kg} / \mathrm{m}^{2}$, respectively. Demographics and rate of patients with previous surgery were comparable preoperatively in the two groups. Mean operative time was $92.1 \pm 19.3 \mathrm{~min}$ and hospital stay was $3.4 \pm 0.6$ days as per our standard protocol of discharge. Uneventful postoperative course was recorded in $93 \%$ of patients and only one case of staple line leak was registered in the first 50 cases (group 1). No statistical difference in BMI and \%TWL was found between the two groups at any time of follow-up. Comparison between two groups showed a significant reduction in hospital stay and operative time after the first 50 LSGs $(p<0.05)$. LSG can be performed by newly trained surgeons proctored by senior tutors. At least 50 cases are needed to meet global benchmark cut-offs and few more cases may be required to reach the plateau of the learning curve.
\end{abstract}

Keywords Sleeve gastrectomy $\cdot$ Surgical training $\cdot$ Learning curve $\cdot$ Global benchmarks $\cdot$ Complications

\section{Introduction}

Laparoscopic sleeve gastrectomy (LSG) is currently the most commonly performed procedure worldwide [1] and main reason of its rapid success is the laparoscopic feasibility [2].

Antonio Vitiello

antoniovitiello_@hotmail.it

Giovanna Berardi

giovannaberardi88@gmail.com

Nunzio Velotti

nunzio.velotti@gmail.com

Vincenzo Schiavone

vincenzoschiavone92@gmail.com

Mario Musella

mario.musella@unina.it

1 Advanced Biomedical Sciences Department, Naples

"Federico II" University, AOU "Federico II", Via S. Pansini

5, 80131 Naples, Italy
LSG was initially introduced by Marceau [3] as first part of the duodenal switch operation to preserve vagal innervation and pyloric function. Subsequently, Gagner [4] proposed a staged procedure also for gastric bypass and LSG was the first step, mostly to overcome the challenge of laparoscopic bariatric surgery in patients with BMI $>60 \mathrm{~kg} / \mathrm{m}^{2}$. Since postoperative outcomes demonstrated low morbidity and satisfactory weight loss, LSG achieved the status of a standalone intervention [5].

Despite this feasibility, even for LSG an appropriate learning curve (LC) is mandatory for newly trained surgeons to reduce perioperative complications [6, 7]. Previous studies have proposed a minimum number of 100 cases to reach a significant reduction in operative time and morbidity after Roux-en-Y Gastric Bypass (RYGB) [8, 9], while a shorter LC has been reported for LSG [10,11]. However, the precise number of LSGs required to achieve optimal results is still matter of debate.

Recently, global benchmarks for LSG and RYGB were set as the 75th percentile of morbidity in 19 high-volume 
academic centres in 3 continents: below this value perioperative outcomes are considered acceptable [12].

Aim of this study was to evaluate whether a newly trained laparoscopic surgeon can complete the LC for LSG after 50 cases by comparing perioperative outcomes to recently introduced global benchmarks.

\section{Methods}

Data of first 100 patients undergoing LSG under a newly trained laparoscopic surgeon at our teaching university hospital (caseload $>150$ per year; annual number of publications $>4$ ) were retrospectively collected and included in this study.

Indications for surgery followed the recommendations of the International Federation of Surgery for Obesity (IFSO) [13].

Since the main inclusion criteria was the chronological order, also patients with a body mass index (BMI) $>50 \mathrm{~kg} /$ $\mathrm{m}^{2}$ or with a previous history of bariatric or abdominal surgery were included.

All the procedures were performed by the same surgeon who had recently ( $<1$ year) ended his residency in general surgery. LC of the novel surgeon was assessed using the CUSUM analysis and divided in two groups (Group 1 and 2) of 50 consecutive patients each and perioperative outcomes were compared to the abovementioned global benchmarks.

Data on preoperative demographics (gender, age, related comorbidities, body mass index-BMI and history of previous bariatric surgery, number of superobese subjects), perioperative data (operative time, conversion to open, use of staple-line reinforcement, reoperation rate, length of hospital stay, readmissions, intra- and post-operative complications, mortality) were registered. Weight loss was calculated at 1 , 3 and 6 months as change of BMI and percentage of total weight loss (\%TWL) using the following formula:

[initial weight - final weight/initial weight $] \times 100$.

Postoperative complications were classified in accordance with the Clavien-Dindo classification [14].

Cases requiring supervision from a senior expert surgeon were also recorded and compared.

The present research was approved by the institutional review board of our Department and informed consent was obtained from all patients before surgery.

\section{Surgical technique}

Standard technique for LSG has been previously reported [15-17]: a five trocars approach $(3 \times 12 \mathrm{~mm}, 2 \times 5 \mathrm{~mm})$ was used. The gastrectomy started $2-4 \mathrm{~cm}$ from the pylorus over a 38-40 French bougie. Staple line reinforcements or oversewing are not routinely used at our Institution. The nasogastric tube was removed the day after surgery and an abdominal drain was left along the staple line. A liquid diet was started on postoperative day 3 and discharge was scheduled in case of no clinical signs of leak or stenosis.

\section{Statistical analysis}

Data are expressed as mean \pm DS. Two-tailored $t$ test was used to compare continuous variables as appropriate, while categorical data were compared using the Chi-square or Fisher's exact test. Significant $p$ value was set below 0.05 .

A cumulative sum (CUSUM) analysis was performed for operative time and hospital stay [18, 19]. Predefined limits were set. Results were presented in CUSUM charts which are a graphical presentation of the outcomes of a series of consecutive procedures. During the LC, the CUSUM curve runs above a decision interval when an operation is performed at an unacceptable level. The intervals were set according to global benchmark values (duration of the operation $=90 \mathrm{~min}$; hospital stay $=3$ ).

\section{Results}

\section{Baseline characteristics}

One hundred consecutive patients were included in the present study; female/male ratio was 16/84 and mean preoperative age and BMI were $41.8 \pm 10.3$ years and $42.9 \pm 5.4 \mathrm{~kg} /$ $\mathrm{m}^{2}$ respectively. Eight subjects had previously undergone a bariatric procedure (gastric band) and 18 had a precedent history of abdominal surgery (10 caesarean sections, 4 laparoscopic cholecystectomy, 2 umbilical hernia repairs, 2 appendectomy). Demographics and rate of patients with antecedent surgery were comparable preoperatively in the two groups (Table 1).

\section{Weight loss}

Mean BMI at 1, 3 and 6 months after LSG was $38.2 \pm 4.9$, $35 \pm 4.9$ and $30 \pm 6$ respectively. Percentage of total weight loss $(\% \mathrm{TWL})$ was $10.3 \pm 4.5$ after 1 month, $17.9 \pm 6.1$ at 3 months and $27.6 \pm 11.2$ after 6 months. No statistical difference in BMI and \%TWL was found between the two groups at any time of follow-up (Figs. 1, 2). Follow-up rate at 1,3 and 6 months were $100 \%, 100 \%$ and $98 \%$ in group 1 and $100 \%, 96 \%$ and $82 \%$ in group 2 . 
Table 1 Comparison of demographics in the two groups of patients

\begin{tabular}{llll}
\hline Parameter & Group 1 $(n=50)$ & Group 2 $(n=50)$ & $p$ value \\
\hline Age (years) & $41.6 \pm 10.6$ & $42 \pm 10$ & 0.19 \\
Sex (F/M) & $7 / 43$ & $9 / 41$ & 0.29 \\
BMI $\left(\mathrm{kg} / \mathrm{m}^{2}\right)$ & $43.6 \pm 5.8$ & $42.2 \pm 4.8$ & 0.71 \\
Previous bariatric surgery $(n, \%)$ & $5(10 \%)$ & $3(6 \%)$ & 0.71 \\
Previous abdominal surgery $(n, \%)$ & $10(20 \%)$ & $8(16 \%)$ & 0.60 \\
Staple line reinforcement/oversewing & $2(4 \%)$ & $1(2 \%)$ & 1 \\
Patients with BMI $>50$ & $6(12 \%)$ & $3(6 \%)$ & 0.29 \\
\hline
\end{tabular}

Group $1=\mathrm{LSG}$ cases from 1 to 50 ; Group $2=\mathrm{LSG}$ cases from 51 to 100

Italic values indicate statistically significant $p$ values $(p<0.05)$

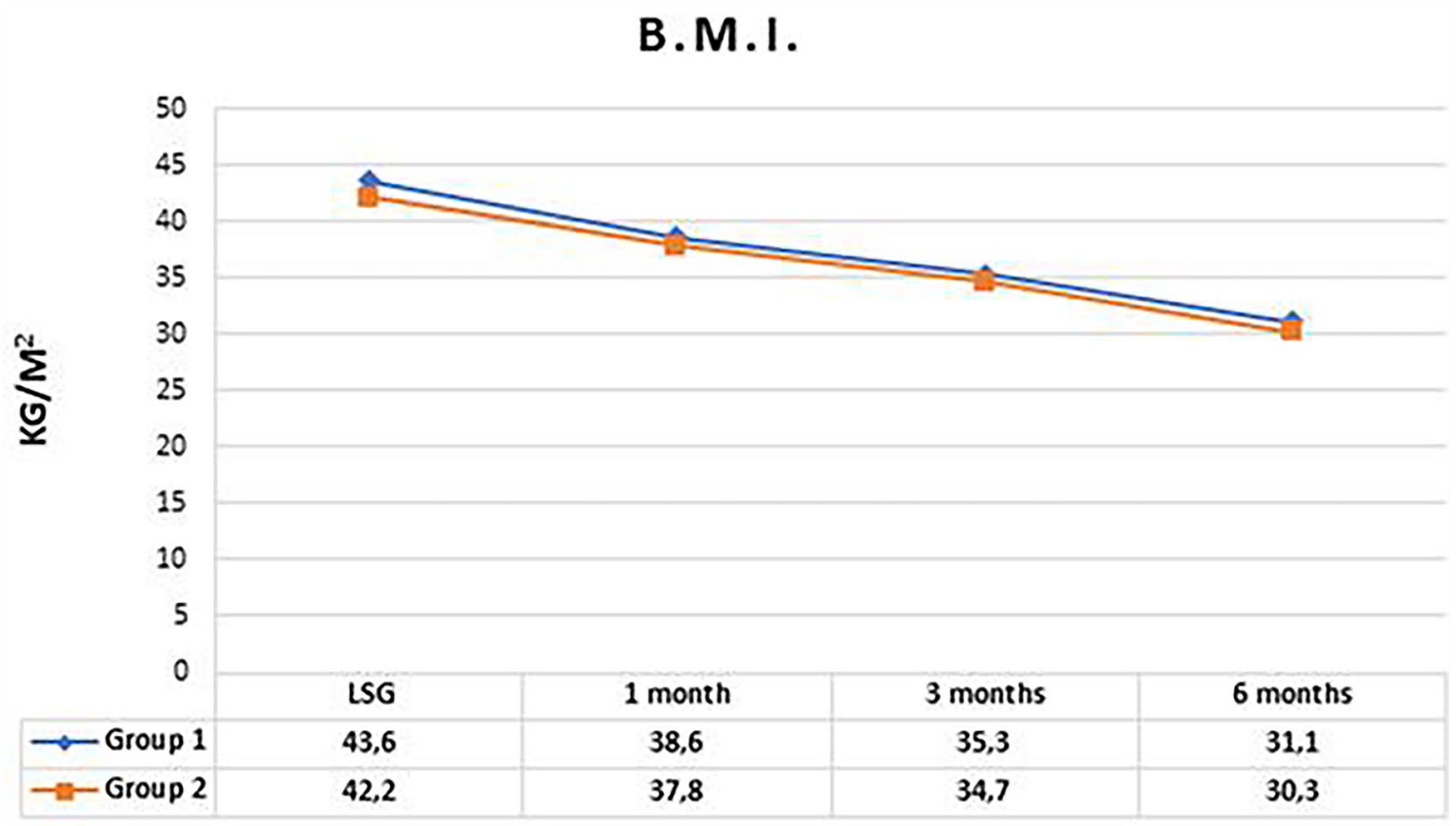

Fig. 1 Trend of BMI in the two groups in the first 6 months. $p$ value was $0.41,0.58$ and 0.48 respectively

\section{Complications and comparison with global benchmarks}

Mean operative time was $92.1 \pm 19.3 \mathrm{~min}$ and hospital stay was $3.4 \pm 0.6$ days as per our standard protocol of discharge. Four patients had postoperative bleeding requiring transfusion and one subjects was readmitted due to hypokalaemia caused by induced vomit. Only one case of staple line leak was registered in the first 50 cases (group 1) and was successfully treated with oesophageal stenting. Uneventful postoperative course was recorded in $93 \%$ of patients. Comparison between two groups shows a significant reduction in hospital stay and operative time after the first 50 LSGs ( $p<0.05$, Tables 2 and 3$)$. As reported in Tables 2 and 3, all benchmark values, except for bleeding, were satisfied in group 2 .
Cases requiring supervision were 7 (14\%) in group 1 and 2 $(4 \%)$ in group $2(p<0.15)$.

\section{CUSUM analysis of the learning curve}

CUSUM graph of the operative time (Fig. 3) runs above the predetermined limit till the 40th cases but reaches the plateau after the 62 nd operation.

CUSUM graph of the hospital stay (Fig. 4) runs above the accepted limit (due to our protocol) but there is a significant slope after the 70 th case. 


\section{(\%TWL)}

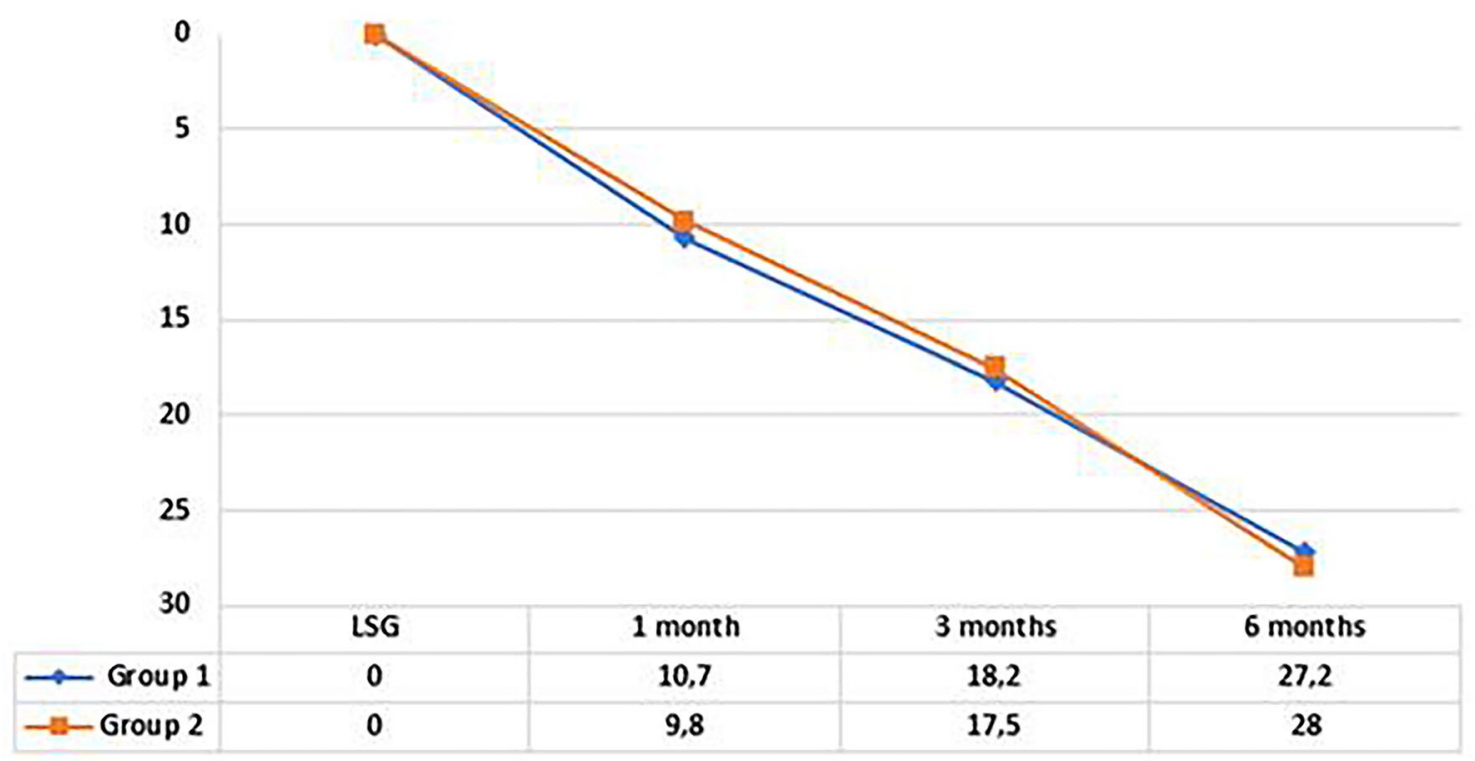

Fig. 2 Trend of \%TWL in the two groups in the first 6 months. $p$ value was $0.36,0.57$ and 0.73 respectively

Table 2 Comparison of perioperative complications in the two groups with global benchmarks

Table 3 Comparison of postoperative complications ( $<90$ days) in the two groups with global benchmarks

\begin{tabular}{lllll}
\hline Perioperative complications & $\begin{array}{l}\text { Benchmark cutoffs } \\
\text { (75th Percentile) }\end{array}$ & Group 1 & Group 2 & $p$ value \\
\hline Operation duration (min) & 90 & $97.1 \pm 21$ & $87.1 \pm 16$ & 0.009 \\
Conversion to open & $0 \%$ & 0 & 0 & 1 \\
Intraoperative blood transfusion & $0 \%$ & 0 & 0 & 1 \\
Postoperative blood transfusion & $1.3 \%$ & $(3) 6 \%$ & $(1) 2 \%$ & 0.6 \\
Postoperative ICU admission & $0 \%$ & $(1) 2 \%$ & $0 \%$ & 1 \\
ICU stay in patients admitted to ICU (days) & 4 & 2 & 0 & $n / a$ \\
Hospital stay & 3 & $3.6 \pm 0.67$ & $3.3 \pm 0.5$ & 0.03 \\
\hline
\end{tabular}

Italic values indicate statistically significant $p$ values $(p<0.05)$

\begin{tabular}{lllll}
\hline Perioperative complications until 90 days & $\begin{array}{l}\text { Benchmark cutoffs } \\
\text { (75th Percentile) }\end{array}$ & Group 1 & Group 2 & $p$ value \\
\hline Uneventful postoperative course & $>88 \%$ & $(44) 88 \%$ & $(49) 98 \%$ & 0.11 \\
Readmission & $<5.5 \%$ & $1(2 \%)$ & $(0) 0 \%$ & 1 \\
Reoperation & $<3 \%$ & $0 \%$ & $0 \%$ & 1 \\
Any complication & $<12 \%$ & $(6) 12 \%$ & $1(2 \%)$ & 0.11 \\
Complication grade> IIIa & $<5.5 \%$ & $0 \%$ & $0 \%$ & 1 \\
Mortality & $0 \%$ & $0 \%$ & $0 \%$ & 1 \\
Staple line leak & $<0.15 \%$ & $1(2 \%)$ & $(0) 0 \%$ & 1 \\
Dysphagia/Stenosis of the gastric tube & $<0.27 \%$ & $0 \%$ & $0 \%$ & 1 \\
Postoperative bleeding & $<1.7 \%$ & $(3) 6 \%$ & $(1) 2 \%$ & 0.6 \\
Small bowel obstruction & $0 \%$ & $0 \%$ & $0 \%$ & 1 \\
Wound infection & $0 \%$ & $0 \%$ & $0 \%$ & 1 \\
\hline
\end{tabular}

Italic values indicate statistically significant $p$ values $(p<0.05)$ 
Updates in Surgery (2021) 73:1891-1898

1895

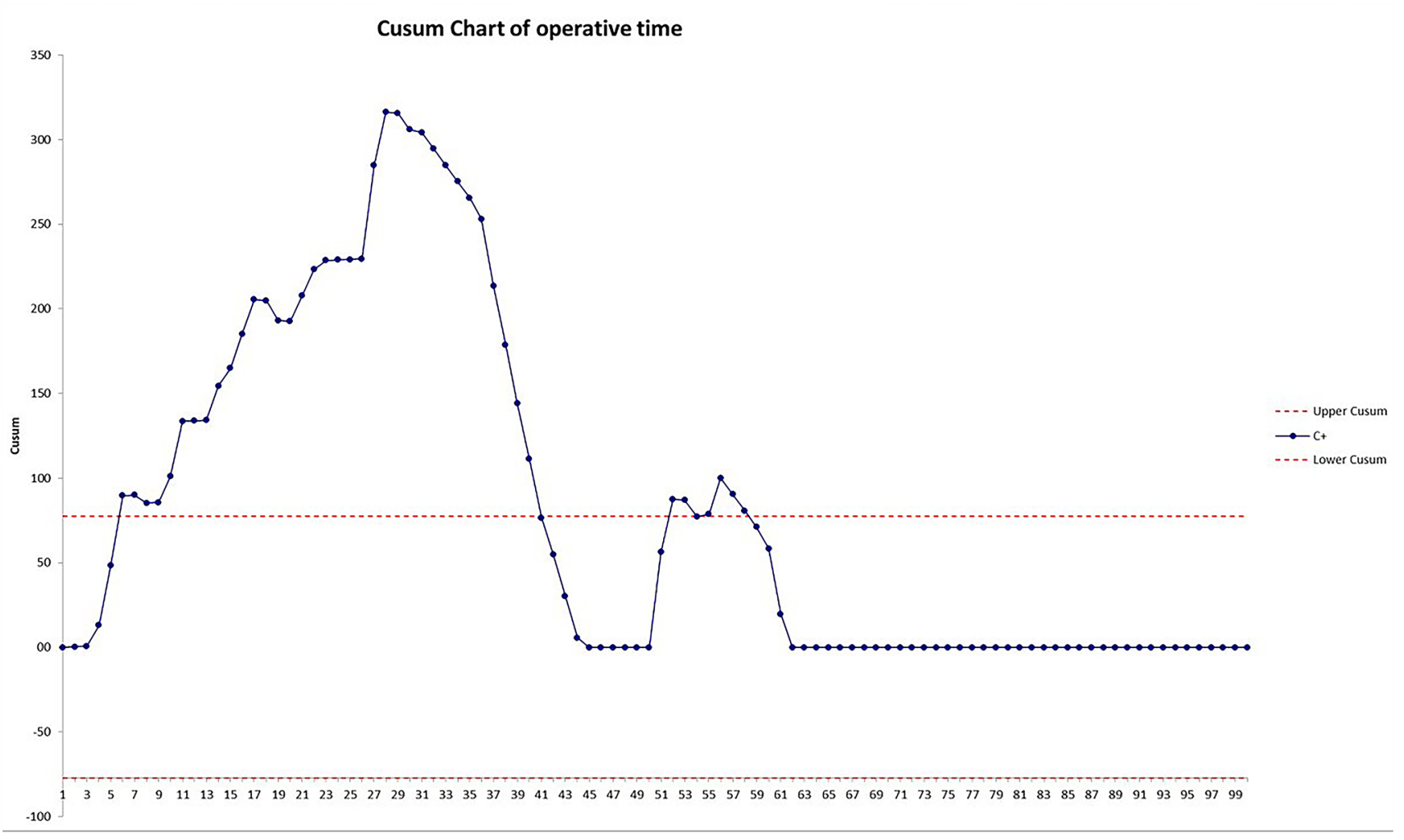

Fig. 3 CUSUM curve of operative time

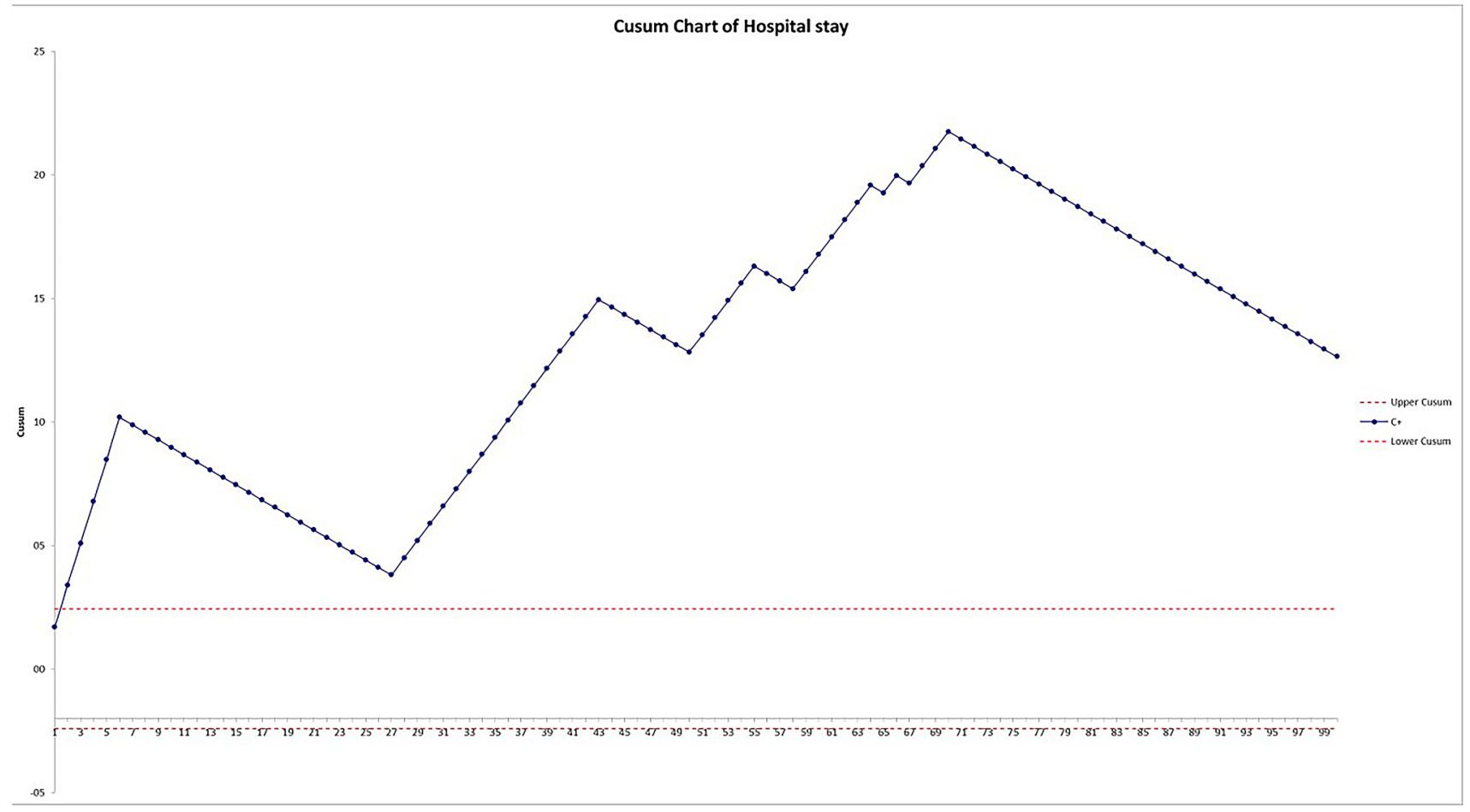

Fig. 4 CUSUM curve of hospital stay

Springer 


\section{Discussion}

Training in surgery is a continuous journey that never really ends. However, optimal operative performance can be achieved for every intervention after a certain number of procedures. There are two main methods to determine whether adequate expertise has been reached: one is to compare perioperative outcomes with international standard values; the second is to statistically and graphically analyse results with the CUSUM calculation. A recent article has demonstrated that global benchmarks [12] are useful indicators for the learning curve of the RYGB and our experience suggest that those values are applicable also for LSG.

Previous experiences have demonstrated that perioperative outcomes of laparoscopic gastrectomies for cancer [20] or for bariatric purpose ${ }^{11}$ significantly improve after the first 100 cases. In a study by Zachariah et al. [21], post-operative morbidity after LSG significantly decreased after the first 50 cases, while Prevot et al. [22] showed a significant difference in terms of operative time and weight loss after 28 cases.

On the contrary, other papers [23,24] have reported that a longer case series ( $>500$ procedures) is necessary for RYGB. Current training of the American Society for Metabolic and Bariatric Surgery (ASMBS) requires fellows to participate in at least 100 weight-loss operations, of which at least 50 should be gastric bypass interventions [25].

Nevertheless, many factors may influence peri-operative outcomes of bariatric surgery such as previous non-bariatric surgical experience, individual skills, careful selection of patients, adequate mentoring by a senior tutor and the support of a multidisciplinary team of a high-volume centre.

Several articles have indeed proved that both LSG and RYGB can be safely performed in structured teaching program by trainee in an early stage of surgical education without untoward consequences for the patient [26, 27].

Despite these enthusiastic reports, it is undeniable that bariatric surgery required advanced laparoscopic skills, without which worrisome complications such as staple line leak, bleeding or stenosis may occur [28-32]. A recent prospective study involving 50 general surgery residency programs in the United States showed that meaningful autonomy for LSG was achieved only by $45 \%$ of the trainees in the fifth postgraduate year [33].

Our experience shows that, even if outcomes of first cases of a newly trained surgeon satisfy most benchmark cut-offs, CUSUM analyses demonstrates that a longer series of procedures is needed to reach the plateau of the learning curve. Indeed, all benchmark criteria were met in group 2, except for bleeding, but on this matter, we should consider that all postoperative haemorrhages were resolved without reoperation.
Significant shorter hospital stay was recorded in group 2 and CUSUM graph shows a plateau after the 70th case. Surgeon's confidence could be the reason of this earlier discharge rather than his proficiency; however, this further demonstrates that at least 50 cases are needed to become confident with LSG.

As described above, our discharge protocol provides liquid diet on postoperative day 3 , therefore shorter length of stay cannot be achieved in our department. We are well aware that there is a current discussion on the safety of LSG as a day case surgery [34], but this management of bariatric surgery does not apply to our hospital due to the absence of an accident and emergency unit.

Our data appear even more impressive if we take into account that these global cut-offs were defined including only patients without previous abdominal surgery and excluding superobese subjects, while we did not adopt these safety criteria.

Correct surgical technique is also important to obtain satisfactory weight loss; there is a consensus [35] that sleeve should be fashioned over an orogastric tube of at least $36 \mathrm{Fr}$ starting within $4-5 \mathrm{~cm}$ from the pylorus to avoid leaving behind a large antrum. Subsequently, a large sleeve may be the main cause of insufficient weight loss. Since most of patients in group 2 have undergone surgery less than a year before this paper, we have focused on short term weight loss, which is an important predictor of long-term results [36]. Percentage of total weight loss was greater than $25 \%$ after 6 months without any difference between the two groups, meaning that the stomach was correctly shaped also in the first cases.

No information was collected on obesity related diseases because six months are not enough to document a significant improvement of these conditions and the duration of remission cannot be predicted with such a short period of time.

\section{Conclusion}

Laparoscopic sleeve gastrectomy (LSG) confirms to be a feasible and effective procedure, which can be performed by newly trained surgeons proctored by senior tutors. However, perioperative outcomes within global benchmarks were achieved only after 50 consecutive operations and few more cases may be required to reach the plateau of the learning curve for operative time and hospital stay.

Funding Open access funding provided by Università degli Studi di Napoli Federico II within the CRUI-CARE Agreement. No funding. 


\section{Declarations}

Conflict of interest The authors declare that they have no conflict of interest.

Human participants and/or animals statement All procedures performed in studies involving human participants were in accordance with the ethical standards of the institutional and/or national research committee and with the 1964 Helsinki declaration and its later amendments or comparable ethical standards.

Informed consent Informed consent for LSG was obtained from all individual participants included in the study.

Open Access This article is licensed under a Creative Commons Attribution 4.0 International License, which permits use, sharing, adaptation, distribution and reproduction in any medium or format, as long as you give appropriate credit to the original author(s) and the source, provide a link to the Creative Commons licence, and indicate if changes were made. The images or other third party material in this article are included in the article's Creative Commons licence, unless indicated otherwise in a credit line to the material. If material is not included in the article's Creative Commons licence and your intended use is not permitted by statutory regulation or exceeds the permitted use, you will need to obtain permission directly from the copyright holder. To view a copy of this licence, visit http://creativecommons.org/licenses/by/4.0/.

\section{References}

1. Welbourn R, Hollyman M, Kinsman R, Dixon J, Liem R, Ottosson J, Ramos A, Våge V, Al-Sabah S, Brown W, Cohen R, Walton P, Himpens J (2019) Bariatric surgery worldwide: baseline demographic description and one-year outcomes from the fourth IFSO Global Registry Report 2018. Obes Surg 29(3):782-795. https:// doi.org/10.1007/s11695-018-3593-1 (Epub 2018 Nov 12; PMID: 30421326)

2. Mahawar KK, Omar I, Singhal R, Aggarwal S, Allouch MI, Alsabah SK, Angrisani L, Badiuddin FM, Balibrea JM, Bashir A, Behrens E, Bhatia K, Biertho L, Biter LU, Dargent J, De Luca M, DeMaria E, Elfawal MH, Fried M, Gawdat KA, Graham Y, Herrera MF, Himpens JM, Hussain FA, Kasama K, Kerrigan D, Kow L, Kristinsson J, Kurian M, Liem R, Lutfi RE, Menon V, Miller K, Noel P, Ospanov O, Ozmen MM, Peterli R, Ponce J, Prager G, Prasad A, Raj PP, Rodriguez NR, Rosenthal R, Sakran N, Santos JN, Shabbir A, Shikora SA, Small PK, Taylor CJ, Wang C, Weiner RA, Wylezol M, Yang W, Aminian A (2021) The first modified Delphi consensus statement on sleeve gastrectomy. Surg Endosc. https://doi.org/10.1007/s00464-020-08216-w (Epub ahead of print. PMID: 33433676)

3. Marceau P, Biron S, Bourque RA, Potvin M, Hould FS, Simard S (1993) Biliopancreatic diversion with a new type of gastrectomy. Obes Surg 3(1):29-35. https://doi.org/10.1381/096089293765559 728 (PMID: 10757900)

4. Regan JP, Inabnet WB, Gagner M, Pomp A (2003) Early experience with two-stage laparoscopic Roux-en-Y gastric bypass as an alternative in the super-super obese patient. Obes Surg 13(6):861-864. https://doi.org/10.1381/096089203322618669 (PMID: 14738671)

5. Gumbs AA, Gagner M, Dakin G, Pomp A (2007) Sleeve gastrectomy for morbid obesity. Obes Surg 17(7):962-969. https://doi. org/10.1007/s11695-007-9151-x (PMID: 17894158)

6. Giudicelli G, Diana M, Chevallay M, Blaser B, Darbellay C, Guarino L, Jung MK, Worreth M, Gero D, Saadi A (2020) Global benchmark values for laparoscopic roux-en-y-gastric bypass: a potential new indicator of the surgical learning curve. Obes Surg. https://doi.org/10.1007/s11695-020-05030-0 (Epub ahead of print. PMID: 33048287)

7. Schauer PR, Ikramuddin S (2001) Laparoscopic surgery for morbid obesity. Surg Clin N Am 81(5):1145-1179

8. Shikora SA, Kim JJ, Tarnoff ME et al (2005) Laparoscopic Rouxen-y gastric bypass: results and learning curve of a high-volume academic program. Arch Surg 140(4):362-367

9. Schauer P, Ikramuddin S, Hamad G et al (2003) The learning curve for laparoscopic roux-en-y gastric bypass is 100 cases. Surg Endosc 17(2):212-215

10. Zacharoulis D, Sioka E, Papamargaritis D, Lazoura O, Rountas C, Zachari E, Tzovaras G (2012) Influence of the learning curve on safety and efficiency of laparoscopic sleeve gastrectomy. Obes Surg 22:411-415

11. Casella G, Soricelli E, Giannotti D, Bernieri MG, Genco A, Basso N, Redler A (2016) Learning curve for laparoscopic sleeve gastrectomy: role of training in a high-volume bariatric center. Surg Endosc 30(9):3741-3748. https://doi.org/10.1007/s00464-0154670-3 (Epub 2015 Dec 16; PMID: 26675935)

12. Gero D, Raptis DA, Vleeschouwers W, van Veldhuisen SL, Martin AS, Xiao Y, Galvao M, Giorgi M, Benois M, Espinoza F, Hollyman M, Lloyd A, Hosa H, Schmidt H, Garcia-Galocha JL, van de Vrande S, Chiappetta S, Menzo EL, Aboud CM, Lüthy SG, Orchard P, Rothe S, Prager G, Pournaras DJ, Cohen R, Rosenthal R, Weiner R, Himpens J, Torres A, Higa K, Welbourn R, Berry M, Boza C, Iannelli A, Vithiananthan S, Ramos A, Olbers T, Sepúlveda M, Hazebroek EJ, Dillemans B, Staiger RD, Puhan MA, Peterli R, Bueter M (2019) Defining global benchmarks in bariatric surgery: a retrospective multicenter analysis of minimally invasive Roux-en-Y gastric bypass and sleeve gastrectomy. Ann Surg 270(5):859-867. https://doi.org/10.1097/SLA.0000000000 003512 (PMID: 31592894)

13. Fried M, Yumuk V, Oppert JM et al (2014) Interdisciplinary European guidelines on metabolic and bariatric surgery. Obes Surg 24(1):42-55

14. Dindo D, Demartines N, Clavien P-A (2004) Classification of surgical complications. Ann Surg 240(2):205-213

15. Velotti N, Manigrasso M, Di Lauro K, Vitiello A, Berardi G, Manzolillo D, Anoldo P, Bocchetti A, Milone F, Milone M, De Palma GD, Musella M (2019) Comparison between LigaSure ${ }^{\mathrm{TM}}$ and Harmonic ${ }^{\circledR}$ in laparoscopic sleeve gastrectomy: a single-center experience on 422 patients. J Obes 2019:3402137. https://doi.org/10. 1155/2019/3402137 (PMID:30719344; PMCID:PMC6335858)

16. Vitiello A, Pilone V, Ferraro L, Forestieri P (2018) Is the sleeve gastrectomy always a better procedure? Five-Year results from a retrospective matched case-control study. Obes Surg 28(8):23332338. https://doi.org/10.1007/s11695-018-3161-8 (PMID: 29549660)

17. Vitiello A, Berardi G, Velotti N, De Palma GD, Musella M (2020) Should Sleeve gastrectomy be considered only as a first step in super obese patients? 5-year results from a single center. Surg Laparosc Endosc Percutan Tech. https://doi.org/10.1097/SLE. 0000000000000866 (Epub ahead of print. PMID: 32956334)

18. Morató O, Poves I, Burdío F, Sánchez-Velázquez P, Duran X, Grande L (2020) Evaluation of the learning curve for laparoscopic pancreatoduodenectomy by CUSUM analyses. Cohort study. Int J Surg 80:61-67. https://doi.org/10.1016/j.ijsu.2020.05.009 (Epub 2020 Jul 7; PMID: 32650295)

19. Biau DJ, Williams SM, Schlup MM, Nizard RS, Porcher R (2008) Quantitative and individualized assessment of the learning curve using LC-CUSUM. Br J Surg 95(7):925-929. https://doi.org/10. 1002/bjs.6056 (PMID: 18498126)

20. Priego P, Cuadrado M, Ballestero A, Galindo J, Carda P, Lobo E (2019) Outcomes of the learning curve in our first 100 consecutive 
laparoscopic gastrectomies. Surg Laparosc Endosc Percutan Tech 29(2):126-132. https://doi.org/10.1097/SLE.0000000000000622 (PMID: 30629034)

21. Zachariah SK, Chang PC, Ooi AS, Hsin MC, Kin Wat JY, Huang CK (2013) Laparoscopic sleeve gastrectomy for morbid obesity: 5 years experience from an Asian Center of Excellence. Obes Surg 23:939-946

22. Prevot F, Verhaeghe P, Pequignot A, Rebibo L, Cosse C, Dhahri A, Regimbeau JM (2014) Two lessons from a 5-year follow-up study of laparoscopic sleeve gastrectomy: persistent, relevant weight loss and a short surgical learning curve. Surgery 155:292-299

23. Doumouras AG, Saleh F, Anvari S, Gmora S, Anvari M, Hong D (2018) Mastery in bariatric surgery: the long-term surgeon learning curve of Roux-en-Y gastric bypass. Ann Surg 267(3):489494. https://doi.org/10.1097/SLA.0000000000002180 (PMID: 28230663)

24. El-Kadre L, Tinoco AC, Tinoco RC et al (2013) Overcoming the learning curve of laparoscopic Roux-en-Y gastric bypass: a 12-year experience. Surg Obes Relat Dis 9:867-872

25. https://asmbs.org/app/uploads/2014/05/CoreCurriculumASMBSF ellowshipTraining.pdf

26. van Rijswijk AS, Moes DE, Geubbels N, Hutten BA, Acherman YIZ, van de Laar AW, de Brauw M, Bruin SC (2018) Can a laparoscopic Roux-en-Y gastric bypass be safely performed by surgical residents in a bariatric center-of-excellence? The learning curve of surgical residents in bariatric surgery. Surg Endosc 32(2):1012-1020. https://doi.org/10.1007/s00464-017-5779-3 (Epub 2017 Sep 21; PMID: 28936562)

27. Major P, Wysocki M, Dworak J, Pędziwiatr M, Małczak P, Budzyński A (2017) Are bariatric operations performed by residents safe and efficient? Surg Obes Relat Dis 13(4):614-621. https://doi.org/10.1016/j.soard.2016.11.017 (Epub 2016 Nov 23; PMID: 28159560)

28. Shoar S, Hosseini FS, Gulraiz A, Ikram W, Javed M, Naderan M, Shoar N, Modukuru VR (2020) Intrathoracic gastric fistula after bariatric surgery: a systematic review and pooled analysis. Surg Obes Relat Dis. https://doi.org/10.1016/j.soard.2020.10.030 (Epub ahead of print. PMID: 33334678)

29. Al-Mazrou AM, Cruz MV, Dakin G, Bellorin O, Pomp A, Afaneh C. Stratification of Readmission after Bariatric Surgery by Day of Post-Discharge Presentation. Obes Surg. 2020 doi: https://doi. org/10.1007/s11695-020-05130-x. Epub ahead of print. PMID: 33206297.

30. Musella M, Susa A, Manno E, De Luca M, Greco F, Raffaelli M, Cristiano S, Milone M, Bianco P, Vilardi A, Damiano I, Segato
G, Pedretti L, Giustacchini P, Fico D, Veroux G, Piazza L (2017) Complications following the mini/one anastomosis gastric bypass (MGB/OAGB): a multi-institutional survey on 2678 patients with a mid-term (5 years) follow-up. Obes Surg 27(11):2956-2967. https://doi.org/10.1007/s11695-017-2726-2 (PMID: 28569357)

31. Musella M, Milone M, Bianco P, Maietta P, Galloro G (2016) Acute leaks following laparoscopic sleeve gastrectomy: early surgical repair according to a management algorithm. J Laparoendosc Adv Surg Tech A 26(2):85-91. https://doi.org/10.1089/lap.2015. 0343 (Epub 2015 Dec 15 PMID: 26671482)

32. Marie L, Robert M, Montana L, De Dominicis F, Ezzedine W, Caiazzo R, Fournel L, Mancini A, Kassir R, Boullu S, Barthet M, D'Journo XB, Bège T (2020) A French National Study on gastropleural and gastrobronchial fistulas after bariatric surgery: the impact of therapeutic strategy on healing. Obes Surg 30(8):3111-3118. https://doi.org/10.1007/s11695-020-04655-5 (PMID: 32382962)

33. Bohnen JD, George BC, Zwischenberger JB, Kendrick DE, Meyerson SL, Schuller MC, Fryer JP, Dunnington GL, Petrusa ER, Gee DW (2020) Trainee autonomy in minimally invasive general surgery in the United States: establishing a national benchmark. J Surg Educ 77(6):e52-e62. https://doi.org/10.1016/j.jsurg.2020. 07.033 (Epub 2020 Oct 23; PMID: 33250116)

34. Aryaie AH, Reddy V, Dattilo Z, Janik MR (2021) Safety of sameday discharge after laparoscopic sleeve gastrectomy: propensity score-matched analysis of the Metabolic and Bariatric Surgery Accreditation and Quality Improvement Program Registry. Surg Obes Relat Dis 17(1):46-53. https://doi.org/10.1016/j.soard.2020. 08.039 (Epub 2020 Sep 6; PMID: 33268322)

35. Mahawar KK, Omar I, Singhal R et al (2021) The first modified Delphi consensus statement on sleeve gastrectomy. Surg Endosc. https://doi.org/10.1007/s00464-020-08216-w

36. Wang L, Sang Q, Du D, Zheng X, Lian D, Zhang N (2021) Early weight loss after laparoscopic sleeve gastrectomy predicts sustained weight maintenance among Chinese individuals with a BMI $<35 \mathrm{~kg} / \mathrm{m}^{2}$. Obes Surg. https://doi.org/10.1007/s11695020-05173-0 (Epub ahead of print. PMID: 33392996)

Publisher's Note Springer Nature remains neutral with regard to jurisdictional claims in published maps and institutional affiliations. 